\title{
Phase transitions in asymmetric Potts models: Breakdown of the classical mean-field picture
}

\author{
M. A. Gusmão* \\ Serin Physics Laboratory, Rutgers University, Piscataway, New Jersey 08854
}

(Received 14 August 1986)

\begin{abstract}
It is shown that mean-field theory fails to give a correct qualitative picture of the thermodynamic behavior of the $q$-state Potts model when the exchange interaction is anisotropic in spin space. The correct picture is recovered either by introducing a single-particle anisotropy or by taking correlations into account via a Bethe-Peierls approximation. This analysis helps the interpretation of previous renormalization-group results for asymmetric Potts models.
\end{abstract}

\section{INTRODUCTION}

The Potts model ${ }^{1}$ of a ferromagnet has been extensively studied $^{2}$ either in its original lattice formulation or in the continuum version ${ }^{3-6}$ as a Euclidean $\phi^{3}$ field theory. Extensions of the model have also been introduced ${ }^{7-9}$ that allow for anisotropy in spin space. These are used, for example, to describe structural phase transitions in perovskites. $^{7,8}$

In a recent paper, Barbosa, Gusmão, and Theumann ${ }^{9}$ discussed the phase transitions in the continuum version of the $q$-state Potts model with symmetry breaking, using a form of the renormalization group (RG) suitable for studying the crossover behavior when some components of the order-parameter field remain massive through the transition. ${ }^{10}$

The purpose of this work is to complement the analysis of Ref. 9, where the interpretation of the RG results was based on a mean-field theory (MFT) which, as I shall discuss below, is not appropriate for the anisotropic case. In particular, the MFT predicts the existence of a disordered phase with zero magnetization even when the exchange anisotropy favors only one of the $q$ states against all the others. The existence of such a phase is obviously not expected on physical grounds. This failure can be explained by the fact that an asymmetry in the exchange interaction manifests itself through correlations, which are neglected in MFT. Indeed, when a single-particle (crystal-field) anisotropy is introduced (Sec. III) it appears as an effective magnetic field in the Landau free energy and, consequently, the disordered phase is not present. I also show that in the case of purely exchange asymmetry the introduction of correlations through a simple Bethe-Peierls approximation ${ }^{11}$ (Sec. IV) results in the absence of a disordered phase. This precludes a paramagnetic-to-ferromagnetic second-order phase transition, although a first-order transition between a weakly and a strongly magnetized phase is not ruled out. ${ }^{8}$ It also explains the absence of a nontrivial fixed point of the RG for this case, ${ }^{9}$ since the mean-field minimum at zero-order parameter, around which the perturbation expansion is performed, is no longer a minimum when the asymmetric interaction is taken into account.

A controversial point concerning the continuation of the results for small noninteger $q$ that is important to understand the role of the RG fixed point and critical exponents is also addressed (Sec. V).

\section{THE MODEL AND RG RESULTS}

The Hamiltonian of the $q$-state Potts model in a lattice with only nearest-neighbor interactions is usually ${ }^{2}$ written as

$$
H=-J \sum_{\langle i j\rangle} \delta_{\sigma_{i} \sigma_{j}}
$$

where $\delta_{\sigma_{i} \sigma_{j}}$ is the Kronecker $\delta$ function, $\langle i j\rangle$ denotes a pair of nearest-neighbor sites, and the $\sigma_{i}$ 's can assume $q$ different values. The relationship between this model and the Ising or Heisenberg models can be made explicit by writing down a spin Hamiltonian for it, namely,

$$
H=-J \sum_{\langle i j\rangle} \mathbf{S}_{i} \cdot \mathbf{S}_{j},
$$

where the "spins" $\mathbf{S}_{i}$ can be in any one of the $q$ states described by the position vectors $\mathbf{e}_{r}(r=1, \ldots, q)$ of the vertices of a hypertetrahedron in $n=q-1$ dimensions. ${ }^{3,4}$ Apart from a constant shift in energy, (1) and (2) share the sample spectrum.

The second form of the Potts-model Hamiltonian is more suitable for the introduction of anisotropy. The generalized or asymmetric ${ }^{12}$ Potts model is defined by

$$
H=-\sum_{\langle i j\rangle} \sum_{\alpha=1}^{n} J_{\alpha} S_{i}^{\alpha} S_{j}^{\alpha},
$$

where the $J_{\alpha}$ 's can assume different values for each component of the spins. The continuum version of this model ${ }^{9}$ is described by the Landau-Ginzburg-Wilson Hamiltonian

$$
-\mathcal{H}=\frac{1}{2} \sum_{\alpha} \int d \mathbf{k}\left(k^{2}+m_{\alpha}^{2}\right) \phi_{\alpha}(\mathbf{k}) \phi_{\alpha}(-\mathbf{k})-\frac{1}{3 !} \sum_{\alpha, \beta, \gamma} u_{\alpha \beta \gamma} D_{\alpha \beta \gamma} \int d \mathbf{k} d \mathbf{k}^{\prime} \phi_{\alpha}(\mathbf{k}) \phi_{\beta}\left(\mathbf{k}^{\prime}\right) \phi_{\gamma}\left(-\mathbf{k}-\mathbf{k}^{\prime}\right)+O\left(\phi^{4}\right),
$$

where the tensorial coefficients $D_{\alpha \beta \gamma}$ are defined in terms of the components of the vectors $\mathbf{e}_{r}$ as $D_{\alpha \beta \gamma}=\sum_{r} e_{r}^{\alpha} e_{r}^{\beta} e_{r}^{\gamma}$. 
I will be interested in the case of uniaxial anisotropy. The vectors $e_{r}$ will be chosen in a particular arrangement ${ }^{4}$ so that one of them, say $\mathbf{e}_{1}$, lies entirely on the symmetry axis. It is easier to visualize in the four-state model, in which these vectors define a tetrahedron in threedimensional space. Then $\mathbf{e}_{1}$ is the position vector of the upper vertex, lying entirely on the $z$ axis (here chosen to be the symmetry axis), while $\mathbf{e}_{2}, \mathbf{e}_{3}$, and $\mathbf{e}_{4}$ define the basis vertices and have nonzero projections on the $x y$ plane. It is clear that an anisotropy that favors alignment of the spins parallel to the symmetry axis will favor $\mathbf{e}_{1}$ against all the other states. Following the notation of Ref. 9, I call this "longitudinal ordering," in contrast to "transverse ordering" when the preference is for alignment perpendicular to the symmetry axis, thus favoring the $q-1$ states with nonzero transverse components.

The RG treatment of this model, within an $\epsilon$ expansion for $d=6-\epsilon$ dimensions, has been developed with considerable detail in Ref. 9. I will only state here the results I wish to discuss.

(i) For longitudinal ordering, no stable fixed point has been found. In the study of the crossover under variation of the mass of the noncritical transverse components, ${ }^{10}$ the fixed point was seen to run away from the symmetric value, without crossing over to a finite limit.

(ii) For transverse ordering, variation of the longitudinal mass showed a crossover from the symmetric fixed point to a nontrivial stable fixed point with both the transverse coupling constant and the critical exponents identical to the symmetric case for $q-1$ states.

I will discuss these results in Sec. V. I turn next to the mean-field analysis to show its deficiency in dealing with an interaction asymmetry. Failure to notice this can mislead an interpretation of the $R G$ results based on MFT.

\section{MEAN-FIELD THEORY}

I will start by rewriting the Hamiltonian (3) for the case of uniaxial symmetry, introducing in addition a single-particle anisotropy term:

$$
H=-\sum_{\langle i j\rangle}\left(J_{1} S_{i}{ }^{1} S_{j}^{1}+J_{2} \sum_{\alpha=2}^{n} S_{i}^{\alpha} S_{j}^{\alpha}\right)-D \sum_{i}\left(S_{i}{ }^{1}\right)^{2}
$$

I will consider $J_{1}>J_{2}$ and $D>0$, both conditions favoring longitudinal ordering. Thus, $S_{i}{ }^{1}$ is expected to develop a nonzero average value that $I$ will call $Q$. The corresponding mean-field Hamiltonian is then

$$
H_{\mathrm{MF}}=\frac{1}{2} N z J_{1} Q^{2}-z J_{1} Q \sum_{i} S_{i}{ }^{1}-D \sum_{i}\left(S_{i}^{1}\right)^{2},
$$

where $N$ is the total number of spins and $z$ is the coordination number. It can be seen that there is no information about the transverse coupling in this mean-field Hamiltonian, which for $D=0$ would be exactly the same as in the symmetric case $J_{1}=J_{2}$.

Defining $K_{\alpha}=\beta z J_{\alpha}$ and $g=\beta D$, where $\beta=1 / k_{B} T$, the free energy per particle,

$$
f=\frac{\beta F_{\mathrm{MF}}}{N}=-\frac{1}{N} \ln Z_{\mathrm{MF}}=-\frac{1}{N} \ln \operatorname{Tr} e^{-\beta H_{\mathrm{MF}}},
$$

is given by

$$
f=\frac{1}{2} K_{1} Q^{2}-\ln \sum_{r} \exp \left[K_{1} Q e_{r}^{1}+g\left(e_{r}^{1}\right)^{2}\right] .
$$

To evaluate the last sum I will use the following representation $^{4}$ for the vectors $\mathbf{e}_{r}$ :

$$
e_{r}^{\alpha}=\left(\frac{q(q-1)}{(q-\alpha)(q-\alpha+1)}\right)^{1 / 2} \times\left\{\begin{array}{l}
0 \text { if } r<\alpha, \\
q-\alpha \text { if } r=\alpha, \\
-1 \text { if } r>\alpha,
\end{array}\right.
$$

where the normalization was chosen so that each vector has magnitude $q-1=n$. With this

$$
f=\frac{1}{2} K_{1} Q^{2}-\ln \left(e^{n K_{1} Q+n^{2} g}+n e^{-K_{1} Q+g}\right) .
$$

In order to write it in the form of a Landau free energy the logarithm must be expanded in powers of $Q$. I will also consider the small-anisotropy limit, keeping only terms up to first order in $g$. The result, dropping additive constants and neglecting terms of $O\left(Q^{5}\right)$, is ${ }^{13}$

$$
f=\frac{1}{2} r Q^{2}-\frac{1}{3 !} w Q^{3}+\frac{1}{4 !} u Q^{4}-h Q,
$$

where

$$
\begin{aligned}
h= & n(n-1) K_{1} g, \\
r= & K_{1}\left\{1-n K_{1}\left[1+\left(n^{2}-n+1\right) g\right]\right\}, \\
w= & n(n-1) K_{1}^{3}\left[1+\left(n^{2}-3 n+1\right) g\right], \\
u= & n\left(4 n-n^{2}-1\right) K_{1}^{4} \\
& \quad-n\left(n^{4}-11 n^{3}+15 n^{2}-11 n+1\right) K_{1}^{4} g .
\end{aligned}
$$

Equations (11) and (12) show that the single-particle anisotropy introduces an effective magnetic field in the Landau free energy, thus eliminating the disordered phase. The field term consistently disappears (together with the cubic term) in the Ising case $n=1$.

From a physical point of view, the qualitative picture should be the same for both single-particle and interaction anisotropy. Nevertheless, as we have just seen, MFT does differentiate between the two. Although providing a correct (qualitative) description of the single-particle case, it completely fails to account for interaction asymmetry.

\section{BETHE-PEIERLS APPROXIMATION}

I now turn to a Bethe-Peierls approximation, ${ }^{11}$ which does not neglect correlations, to see if the effect of purely interaction anisotropy is noticeable. In the longitudinal ordering case it should yield the absence of a disordered phase.

The Bethe-Peierls Hamiltonian for a cluster with a central spin $\mathbf{S}_{0}$ and $z$ nearest neighbors is written as

$$
-\beta H_{\mathrm{BP}}=\sum_{i=1}^{z}\left(K_{1} S_{0}^{1} S_{i}{ }^{1}+K_{2} \sum_{\alpha=2}^{n} S_{0}^{\alpha} S_{i}^{\alpha}\right)+h_{0} \sum_{i=1}^{z} S_{i}{ }^{1}+h S_{0}^{1} \text {, }
$$


where $h$ is an applied field (to be set to zero later) and $h_{0}$ is the effective field on the external spins of the cluster. The spontaneous magnetization on sites 0 and $i$ are, respectively, $m_{0}=\left.Z_{\mathrm{BP}}^{-1}\left(\partial Z_{\mathrm{BP}} / \partial h\right)\right|_{h=0}$ and $m_{i}=(1 / z) Z_{\mathrm{BP}}^{-1}$ $\times\left.\left(\partial Z_{\mathrm{BP}} / \partial h_{0}\right)\right|_{h=0}$, where $Z_{\mathrm{BP}}=$ Tre $e^{-\beta H_{\mathrm{BP}}}$ is the partition function. The effective field $h_{0}$ is determined through the consistency condition $m_{0}=m_{i}$.

In order to avoid excessively complicated expressions I will restrict the analysis to the three-state Potts model. The resulting equations for the two magnetizations are then

$$
\begin{aligned}
\frac{1}{2} Z_{\mathrm{BP}} m_{0}= & \left(e^{2\left(h_{0}+2 K_{1}\right)}+2 e^{-\left(h_{0}+2 K_{1}\right)}\right)^{z}-\left(e^{2\left(h_{0}-K_{1}\right)}+2 \cosh \left(3 K_{2}\right) e^{-\left(h_{0}-K_{1}\right)}\right)^{z}, \\
\frac{1}{2} Z_{\mathrm{BP}} m_{i}= & \left(e^{2\left(h_{0}+2 K_{1}\right)}+2 e^{-\left(h_{0}+2 K_{1}\right)}\right)^{z-1}\left(2 e^{2\left(h_{0}+2 K_{1}\right)}-e^{-\left(h_{0}+2 K_{1}\right)}\right) \\
& +2\left[e^{2\left(h_{0}-K_{1}\right)}+2 \cosh \left(3 K_{2}\right) e^{-\left(h_{0}-K_{1}\right)}\right]^{z-1}\left[e^{2\left(h_{0}-K_{1}\right)}-\cosh \left(3 K_{2}\right) e^{-\left(h_{0}-K_{1}\right)}\right] .
\end{aligned}
$$

There is no need to look for a general solution, since the relevant question is whether a disordered phase is present or not. So, the solution to be checked is $m_{0}=m_{i}=0$. This obviously implies $h_{0}=0$ and Eqs. (14) take the form

$$
\begin{aligned}
& \frac{1}{2} Z_{\mathrm{BP}} m_{0}=\left(e^{4 K_{1}}+2 e^{-2 K_{1}}\right)^{z}-\left[e^{-2 K_{1}}+2 \cosh \left(3 K_{2}\right) e^{K_{1}}\right]^{z} \\
& \frac{1}{2} Z_{\mathrm{BP}} m_{i}=\left(e^{4 K_{1}}+2 e^{-2 K_{1}}\right)^{z-1}\left(e^{4 K_{1}}-e^{-2 K_{1}}\right)+2\left[e^{-2 K_{1}}+2 \cosh \left(3 K_{2}\right) e^{K_{1}}\right]^{z-1}\left[e^{-2 K_{1}}-\cosh \left(3 K_{2}\right) e^{K_{1}}\right] .
\end{aligned}
$$

It can be easily checked that the only case in which the right-hand sides of both Eqs. (15) become zero is for $K_{1}=K_{2}$. Thus, there is no disordered phase in the asymmetric case $K_{1} \neq K_{2}$, which is the expected result. Although the calculation was restricted to the three-state model the basic point is present here, namely, the fact that the anisotropy favors one state against the others (in this case two), whose equivalence is maintained.

\section{DISCUSSION}

I have clearly demonstrated the failure of the usual MFT to cope with a situation in which the exchange interaction is anisotropic. In the case of the Potts model, this failure yields the prediction of a disordered phase in the presence of an anistropy that, by favoring one single spin state, rules out the possibility of zero magnetization at finite temperatures. That this failure is related to the neglect of correlations is confirmed by the fact that it can be corrected through the introduction of a single-particle anistropy, or by resorting to an approximation that does not neglect correlations, as the Bethe-Peierls scheme.

The above analysis is relevant for the interpretation of the $R G$ results stated in Sec. II, since the MFT is the classical limit of the effective quantum field theory for the problem. As far as the $\phi^{3}$ theory is concerned, there is no possibility of studying the first-order transition, and the $R G$ deals with fluctuations around the mean-field minimum at $\phi=0$ (a local anisotropy was not considered in the RG treatment of Ref. 9). For the case of longitudinal ordering the asymmetric interaction displaces this minimum, so that no massless fluctuations around $\phi=0$ are possible. This yields the absence of a stable fixed point of the $\mathrm{RG}^{9}{ }^{9}$ For transverse ordering, although the minimum in the (massive) $\phi_{1}$ component is also displaced from zero, the massless transverse fluctuations remain around $\phi=0$ and a fixed point is found ${ }^{9}$ which essentially describes a symmetric system with $q-1$ states. This is also an expected result, since the anisotropy should "freeze out" the state $\mathbf{e}_{1}$, without affecting the equivalence between the remaining $q-1$ states.

The relevant concern here is whether the transverse fixed point and the corresponding critical exponents describe a second-order phase transition or not. I now turn to a discussion of this point for the symmetric theory, since the same conclusions apply for the asymmetric case (transverse ordering) with a shift in the number of states.

Equation (11) for $g=0(h=0)$ is the Landau free energy of the symmetric model (now free of problems of the anisotropic case). The cubic term, which is not present only for $n=1$ (Ising model), is responsible for the existence of two minima and the consequent first-order nature of the phase transition. For $n>1(q>2)$, the large order-parameter minimum occurs for $Q>0$, and it is accepted that the transition is first order. In this case, the fixed point of the $R G$ should describe the spinodal point in the stability limit of the disordered phase. ${ }^{5,6}$ For $n<1$ $(q<2)$, however, the large- $|Q|$ minimum occurs for $Q<0$. The current argument ${ }^{4}$ rules out this minimum as unphysical and considers that the low order-parameter minimum at $Q \gtrsim 0$ is the one that does describe the system. The $R G$ fixed point would then actually describe a second-order phase transition.

The question obviously arising is whether it is possible to simply neglect the unphysical part of the free energy, keeping the rest unaltered, or should the whole theory be revised. Pytte ${ }^{6}$ expressed the hope that the negative- $Q$ minimum would disappear if the Landau free-energy included all orders in $Q$. This, unfortunately, is not the case, as can be seen from Eq. (10), which does include all orders in $Q$. From the minimization condition $\partial f / \partial Q=0$ (and setting $g=0$ ), we obtain

$$
Q=n \frac{e^{(n+1) K Q}-1}{e^{(n+1) K Q+n}} .
$$

A simple inspection of the behavior of the right-hand side of this equation shows that a solution for $Q$ large and negative is always present for $n<1$. There is, however, an important difference between the positive- and negative- $Q$ solutions. Since the limiting value of the right-hand side of (16) is $n$ for $Q>0$ and -1 for $Q<0$, the positive- $Q$ solution always satisfies the condition $|Q| \leq n$ (implied by the normalization of the vectors $\mathbf{e}_{r}$ ), while this condition is violated in the negative- $Q$ solution for $n<1$. This 
may be a mathematical indication of the unphysical nature of this solution.

Pytte $^{6}$ also claims the instability of the negative- $Q$ minimum against transverse fluctuations. What actually happens (and this remained hidden in Pytte's tensorial order parameter) is that the transverse fluctuations become imaginary for $q<2$. This can be understood in terms of the relation $n=q-1$ that makes it impossible to consider transverse fluctuations for $q<2$, when the number of components $n<1$. It appears as imaginary components $e_{r}^{\alpha}$ for $q<\alpha$ in Eq. (9) and yields imaginary tensorial coefficients $D_{\alpha \beta \gamma}$ in Eq. (4).

It is well known and explicitly manifest in the above discussion, that the $q$-state Potts model is not defined for $q<2$. It can only be defined by means of an analytical continuation. So far no such continuation has been clearly achieved, and even the prescription for obtaining the percolation limit ${ }^{14,15} q \rightarrow 1$ is not free of inconsistencies. ${ }^{16}$ Until a mathematically rigorous continuation is worked out, the accepted description of a second-order phase transition in the Potts model for $q<2$ (or asymmetric Potts models for higher values of $q$ ) will remain a conjecture.

\section{ACKNOWLEDGMENTS}

To complete this work I benefited from earlier discussions with Walter Theumann and Marcia Barbosa, and lately with Michael Stephen and Timothy Ziman, whom I also thank for a critical reading of the manuscript. This work was supported in part by Conselho Nacional de Desenvolvimento Cientifico e Tecnologico, Brazil, and by the National Science Foundation under Grant No. DMR-85-20190.
*Permanent address: Instituto de Fisica, Universidade Federal do Rio Grande do Sul, 90049 Porto Alegre, Rio Grande do Sul, Brazil.

${ }^{1}$ R. B. Potts, Proc. Cambridge Philos. Soc. 48, 106 (1952).

${ }^{2}$ For a review, see F. Y. Wu, Rev. Mod. Phys. 54, 235 (1982).

${ }^{3}$ R. P. Zia and D. J. Wallace, J. Phys. A 8, 1495 (1975).

${ }^{4}$ R. G. Priest and T. C. Lubensky, Phys. Rev. B 13, 4159 (1976); 14, 5125(E) (1976).

${ }^{5}$ D. J. Amit, J. Phys. A 9, 1441 (1976).

${ }^{6}$ E. Pytte, Phys. Rev. B 22, 4450 (1980).

${ }^{7}$ D. Blankschtein and A. Aharony, Phys. Rev. B 22, 5549 (1980); J. Phys. C 13, 4635 (1980); 14, 1919 (1981).

${ }^{8}$ J. F. Fontanari and W. K. Theumann, Phys. Rev. B 33, 3530 (1986).

${ }^{9}$ Marcia C. Barbosa, M. A. Gusmão, and W. K. Theumann,
Phys. Rev. B 34, 3165 (1986).

${ }^{10}$ D. J. Amit and Y. Y. Goldschmidt, Ann. Phys. (N.Y.) 114, 356 (1978).

${ }^{11}$ H. A. Bethe, Proc. R. Soc. London Ser. A 150, 552 (1935); R. Peierls, Proc. Cambridge Philos. Soc. 32, 477 (1936).

${ }^{12}$ The term anisotropic is sometimes used to indicate a space anisotropy [see, for example, C. Tsallis, J. Phys. C 15, L757 (1982)]. The denomination asymmetric is probably more suitable for the present case, although I use both terms in the text.

${ }^{13}$ Equation (11) for $h=0$ can also be obtained by taking the classical limit of Eq. (4).

${ }^{14}$ C. M. Fortuin and P. W. Kasteleyn, Physica 57, 536 (1972).

${ }^{15}$ M. J. Stephen, Phys. Rev. B 15, 5674 (1977).

16 J. Rudnick and G. Gaspari, J. Stat. Phys. 42, 833 (1986). 